\title{
Study on Public Service Function of Competitive Sports in Shaanxi and Implementation Path
}

\author{
Ronghua Liu \\ Shaanxi Normal University, Xi'an, Shaanxi, 710119, China
}

\begin{abstract}
Keywords: Competitive sports resource, Public sports service, Shaanxi Province, Function, Implementation path
\end{abstract}

\begin{abstract}
Shaanxi is a sports power in China. Over these years, it has developed sports strongly and especially contributed a lot of sports talents to the state in terms of competitive sports. In recent years, with vigorous publicity and promotion of the slogan of strengthening the country through sports in China, i.e. "carry out national fitness and strengthen the country and people" , sports powers such as Shaanxi Province have been establishing social engineering of supporting public sports service utility with competitive sports resources and expecting to consolidate health construction plan for citizens with public sports resources. This paper discusses public service of competitive sports in Shaanxi Province and main functions and specific path of social public service of competitive sports in terms of existing resources and potential resources of competitive sports in Shaanxi Province.
\end{abstract}

\section{Introduction}

Social public service utility of competitive sports is a path for China to balance competitive sports and mass sports and develop towards sports power from great sports nation. In recent years, Shaanxi Province has been committed to the development of social public service of competitive sports and expected to enrich and manage social mass sports and school sports with rich competitive sports resources and lay a solid foundation for the promotion of competitive sports in the province and even in China.

\section{Necessity of construction of public sports service and social public service utility of competitive sports}

Connotations of public sports service. Contents of public sports service include the provision of a lot of public sports products and services for the purpose of benefiting the society and citizens and establishing future strong environmental atmosphere for mass sports and even the foundation of sports power, including the strengthening of public sports facilities, public sports utility and public sports information. Public sports service can provide richer and more favorable social guarantee for the public in the process of participation in sports activities and create favorable conditions for the participation of more people in sports activities. Therefore, public sports service should be constructed and consumed jointly by the whole society.

Necessity of social public service utility of competitive sports. Competitive sports is a social activity. Therefore, it requires the participation of each social member and even social culture to form certain mass base. In China, the development balance of competitive sports and mass sports has become the most important social issue since the $21^{\text {st }}$ century. In particular, Chinese competitive sports under the whole nation system has gathered the force of all people and accumulated a lot of competitive sports resources. Therefore, it is a feasible program to transform these resources with classification and realize the new path of transforming competitive sports resources into public sports service in the future development and coordination process of competitive sports in China.

\section{Functional analysis on social public service utility of competitive sports}

Social public service utility of competitive sports mainly uses the leading and driving force formed by leading development of competitive sports and drives social public sports service. It 
benefits from social forces. Such regurgitation-feeding behavior for the society has profound significance for strengthening foundation works of Chinese competitive sports - social public sports.

Strengthen the construction of sports foundation works. The use of competitive sports resources to promote social public sports service system is a process to consolidate the construction of sports foundation works. It is mainly manifested in five aspects: first, guiding services brought by competitive sports resources to the society. Such resources mainly refer to skilled talents of competitive sports who enter social communities to help the masses, participate in their sports activities and publicize sports activities. Second, informationalized service of competitive sports resources. Third, service of competitive sports activities. Forth, Shaanxi has complete sports service facilities but their functionality is unsatisfactory. Therefore, Shaanxi must attach importance to the construction and development of sports foundation facilities in the future. Finally, the construction in terms of the organization of public sports service should be based on diversified and multi-level structure and provide guarantee. Therefore, Sports Department and the government of Shaanxi has enhanced the cooperation in function so as to complement each other in abilities, form a competitive sports reform institution with forces of the government, society and market, drive public sports service functions of the government at each level while developing functional characteristics of competitive sports, exert the organization function of grass roots and enable all people to enjoy the welfare of social public sports service. The purpose is to expand Chinese sports population and achieve the final effect of popularization of sports in the society.

Improve the demand degree of expansion of diversified competitive sports culture. Competitive sports has multiple connotations, such as sports event culture. Shaanxi people like football and basketball. Therefore, sports event culture is implemented mainly according to the two sports events. Social public sports service utility rendered by competitive sports is richer in terms of service selection due to the reaction of the masses to different sports cultures derived in the society, such as photography and songs. Capturing the beauty of power and speed of athletes with camera on the arena and manifesting the thought of "higher, faster and stronger" brought by competitive sports can stimulate the fighting spirit of people learnt from sports and allow them to deeply feel the great charm of competitive sports.

Artistic rendering brought by competitive sports resources is more prominent in movie song entertainment industry. While appreciating screen culture works containing elements of competitive sports, people are receiving such sports culture service. It has improved the overall accomplishment of Chinese citizens and met popular demands for cultural diversity. Moreover, the publicity of service and culture through communication media has stimulated the spirit of innovation of competitive sports utility and indirectly laid a foundation for the establishment of Chinese public sports service system ${ }^{[3]}$.

Triune competitive sports coverage. Another advantage of the transformation of competitive sports resources into public sports service function is the formation of triune sports service development organization type in the society. For example, it not only enhances professional competitive sports utility, but also enriches the development of amateur competitive sports utility. For example, sports venues in colleges and universities in Shaanxi are open to the public. This practice has actually realized great-leap-forward development progress of public sports utility, improved complementary laws and regulations of mass sports through the opening of school competitive sports resources, integrated sports and education and increased the utilization and opening proportion of school competitive sports resources. It also strengthens the supply capacity of social public sports service and meets demands of the masses for public sports service ${ }^{[4]}$.

\section{Study on competitive sports resources in Shaanxi Province}

Shaanxi has rich sports resources, including various types of existing resources and potential resources to be exploited. They are important sources of social public sports service utility. 
Analysis on existing sports resources. In Shaanxi, existing sports resources mainly include four medium and large sports venues, including national-level sports venues such as Shaanxi Olympic Sports Center and Shaanxi Gym. They can not only meet requirements of national and international competitions, but also meet requirements of public service open to the public at ordinary times. There are over 8 rebuilt venues such as Shaanxi Olympic Water Park, which form rich social public sports service institutions in Shaanxi.

In addition, Shaanxi has solid reserve of talents in terms of public sports service talents. As there are a galaxy of sports talents in Shaanxi, many high-level athletes do not have the opportunity to attend international competitions on behalf of the country. The province has provided athletes about to retire due to some factors such as injury, disease and age with opportunities for contributing to social public sports service utility again, e.g. cultivate them as high-level coaches, PE teachers and administrative staff in sports organizations, thus maintaining the future development prospect of high-level sports talents and realizing full transformation of high-level sports talents resources into social public sports service utility. Meanwhile, a large batch of sports injury rescue workers and physique test personnel are fundamental resources exploited in public sports service in Shaanxi. They provide more professional safety guarantee for the participation of the masses in sports activities ${ }^{[5]}$.

Analysis on potential sports resources. Shaanxi has unique advantages in potential sports resources such as favorable natural environment of Qinling Mountains. It is the most appropriate place for sports of cyclists. Therefore, Shaanxi has organized Cycling around Qinling Mountains through cooperation with National Sports Bureau, which has attracted domestic and foreign cyclists for participation. For Shaanxi famous for Olympic champions, sports star effect is also an important potential resource for the development of public sports service utility. For example, Olympic champions such as Tian Liang have participated in the publicity of mass sports activities, made a contribution to mass sports and social public sports service utility and popularized sports knowledge and consciousness ${ }^{[6]}$.

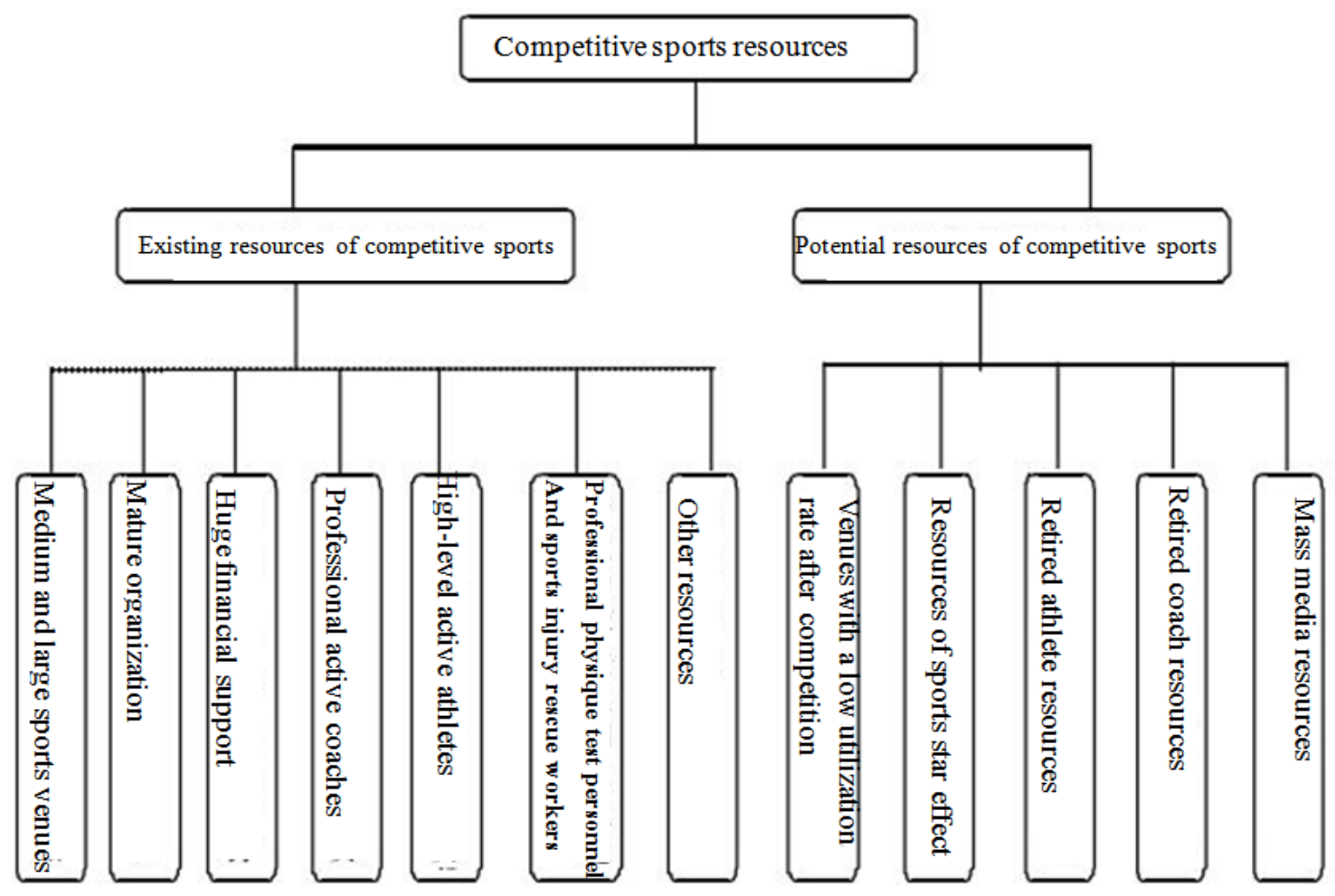

Fig.1. Schematic diagram of classification of competitive sports resources in Shaanxi 


\section{Implementation path of competitive public sports service utility in Shaanxi}

Deep excavation of resources of competitive sports venues. Shaanxi has rich resources of competitive sports venues, but its public function and service development should be improved. It is necessary to conduct deep development and excavation. In particular, the practice of some countries should be followed in terms of the transformation of public service of medium and large competitive sports venues. For example, Sydney Olympic Park in Australia has established cooperative relationship with local sports administration and multiple educational institutions. It provides a research and training place for athletes, judges, coaches and sports doctors, and sets a public open day for surrounding schools at each level. Students can attend sports activities in this famous venue and use all sports resources and services. Therefore, the improvement of comprehensive utilization ratio of sports venues in Shaanxi and deep and free development of their service efficiency are major development objectives to be achieved in public service utility of competitive sports in Shaanxi in the future.

Establishment of characteristic public sports service organization. Public sports service organizations in Shaanxi are not mature. Shaanxi should establish a public sports service organization system from top to bottom with close relation network in the future, make design and overall planning according to requirements for the establishment of public sports service system, promote the coordination and cooperation of social organizations and sports institutions and make public institutions and social non-governmental institutions become main undertakers and beneficiaries of public sports service utility. Therefore, competitive sports resources should be input into public sports service link in the form of organization so as to guarantee mass sports utility better.

Expansion of financing channels of public sports service. Competitive sports resources are important resources of social market development. Therefore, to stabilize the functionality and sustainability of economic public sports service, it is required to input more funds into each field of basic public sports service. According to the current situation, the most feasible program is to optimize and improve the way of financing of Chinese public sports service and expand financing channels in the development process of public service utility of competitive sports. Government-leading publicly offered funds in government allocations, social financing, public investment and personal investment can be integrated organically. Then, financing means can be planned and adjusted according to local financial status of Shaanxi so as to maximize the benefit of economic public sports service utility in Shaanxi.

Establishment of sports health institutions in communities. To guarantee the safety of mass sports, it is required to establish professional health institutions for physique test and sports injury rescue in each community, input competitive sports talents and resources into competitive mass sports and realize health inspection of the masses and popularization of sports and medical science. Such practice can protect the enthusiasm and safety of the masses in their participation in sports activities, improve their common sense of sports indirectly and lay a good quality foundation for the construction of national sports in Shaanxi and even China.

Conclusion: China is in the transformation period of market economy. The development concept of transforming into sports power from great sports nation is moving towards a historical turning point. Therefore, it is vital to implement the construction of public service function utility of competitive sports. According to the current situation, China has a weak foundation of mass sports and low level of public sports service and lacks complete and solid theoretical and practical foundation. Therefore, regions with rich resources of competitive sports such as Shaanxi should exploit potentials of sports service vigorously, optimize excessive resources of competitive sports, fully realize the functionality and realistic value of public service of competitive sports in future development of sports utility and make contributions to the dream of strengthening the country through sports. 


\section{Acknowledgments}

This paper is a philosophical and social science topic of Shaanxi Province. Topic name: Study on Public Service Function of Competitive Sports in Shaanxi and Implementation Path; No. 13P019

\section{References}

[1] Yang Qianping. Study on Establishment of Public Sports Service System - Take Binhai New Area for Example. Nankai University, 2011.24-28.

[2] Ma Huawei, Zhang Kun, Wu Qiong et al. Discussions on Regurgitation-feeding of Competitive Sports for Public Sports Service. Sichuan Sports Science, 2015,(4):109-111.

[3] Dai Xiaoyu, Zhang Kun. Study on Classification and Path in Process of Transformation from Competitive Sports Resources into Public Sports Service. Hubei Sports Science, 2014,(10):865-867.

[4] Shao Kai, Dong Chuansheng. New Discussions on Social Value of Competitive Sports under Strategic Background of National Fitness - Discussions on Public Value of Competitive Sports. Journal of Shandong Sports Institute, 2015,(1):8-13.

[5] Li Zonghao. Interpretations of Competitive Sports and Analysis on Competitive Sports Power. Journal of Tianjin Sports Institute, 2013,28(2):93-95.

[6] Xu Benli. Interpretation and Analysis on Connotations of Strengthening Country through Sports, Competitive Sports and Mass Sports. Journal of Tianjin Sports Institute, 2009,24(2):93-98.

[7] Lu Dongsheng. Development and Application of Modern Science and Technology in Competitive Sports - Comments on Competitive Sports and Leading-edge Science. Contemporary Educational Science, 2015,(14). 